\title{
Certified Transmittance Density Uncertainties for Standard Reference Materials using a Transfer Spectrophotometer
}

J. C. Travis (ret.) M. V. Smith S. J. Choquette Hung-kung Liu

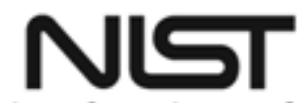

National Institute of Standards and Technology U.S. Department of Commerce

NIST Technical Note 1715 


\section{Certified Transmittance Density Uncertainties for Standard Reference Materials using a Transfer Spectrophotometer}

J. C. Travis (ret.) M. V. Smith

S. J. Choquette

Biochemical Science Division First Operating Unit

Hung-kung Liu Statistical Engineering Division Second Operating Unit

November 2011 
Certain commercial entities, equipment, or materials may be identified in this document in order to describe an experimental procedure or concept adequately. Such identification is not intended to imply recommendation or endorsement by the National Institute of Standards and Technology, nor is it intended to imply that the entities, materials, or equipment are necessarily the best available for the purpose.

National Institute of Standards and Technology Technical Note 1715 Natl. Inst. Stand. Technol. Tech. Note 1715, 13 Pages (November 2011) CODEN: NTNOEF

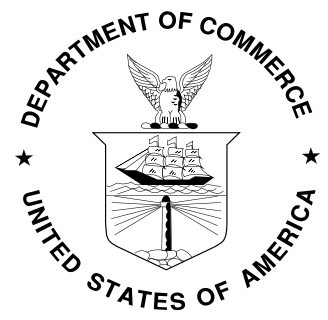




\begin{abstract}
Overall uncertainties are evaluated for the certification of transmittance density (absorbance referred to air) and regular spectral transmittance for solid neutral density filter Standard Reference Materials by means of a transfer spectrophotometer. Traceability is asserted by means of comparison measurements to a recognized reference spectrophotometer. The uncertainties are evaluated without bias correction, using the combination of the legacy combined standard uncertainty values with uncertainty components for the measured bias, the standard uncertainty of this measured bias, and the standard uncertainty characteristic of simple replication for a single measurement using the transfer spectrophotometer. Numerical results are given, and are anticipated to be the values initially quoted on certificates and in reports of recertification. However, these values are subject to change upon future adjustment of estimated uncertainty components for the filters or changes in the observed bias with future determinations.
\end{abstract}

\title{
Key Words
}

Absorbance, bias, certification, spectrophotometer, traceability, transmittance, transmittance density, uncertainty 


\section{Table of Contents}

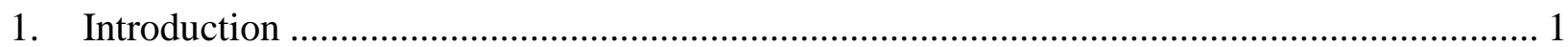

2. Certification Measurements with the Transfer Spectrophotometer...................................... 1

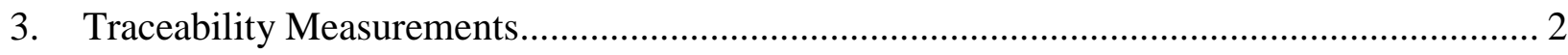

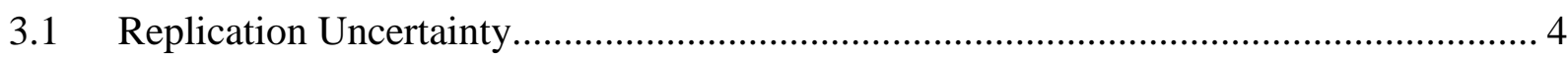

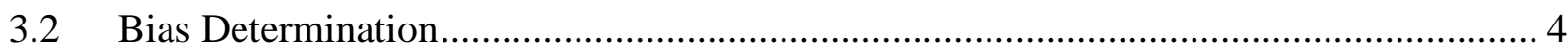

3.3 Transfer Spectrophotometer Uncertainties...................................................... 5

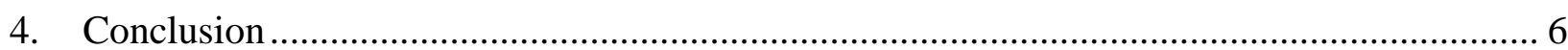

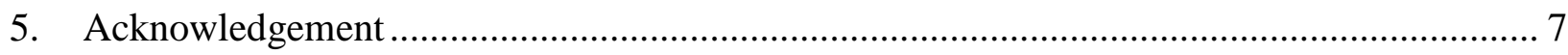

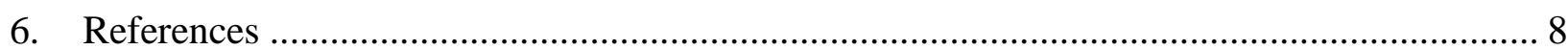




\section{Glossary}

Absorbance, $A$ : Defined from the internal transmittance, $T_{i n t}$, as $A=-\log \left(T_{\text {int }}\right)=\log \left(1 / T_{\text {int }}\right)$.

Bias, $\delta$ : The difference in the reported value of a measurement and the best available estimate of the true value. In the present case, the bias reported is the difference in the transmittance density determined with the transfer spectrophotometer and the reference spectrophotometer.

Combined standard uncertainty, $u_{c}$ : The result of propagating all of the standard uncertainty components of the input quantities for a measurement to yield an uncertainty estimate for a measurement which is the equivalent of a standard deviation, or, approximately, the half width of a $67 \%$ confidence interval.

Expanded uncertainty, $U_{95}$ : Multiplication of the combined standard uncertainty by an "expansion factor" $\mathrm{k} \geq 2$ yields an uncertainty estimate corresponding to, approximately, the half width of a $95 \%$ confidence interval for a measurement. The value $\mathrm{k}=2$ is used in the present instance, reflecting the reasonably large effective degrees of freedom for the evaluation.

Internal transmittance, $T_{\text {int }}$ : The portion of the overall transmittance determined by the absorption of light in the interior of the sample, between the reflective entry and exit faces of a cuvette or solid filter.

Optical density, $O D$ : Defined from the transmittance, $T$, as $O D=-\log (T)=\log (1 / T)$.

Regular Spectral Transmittance, $T(\lambda)$, referred to throughout as transmittance: The ratio of the exit intensity to the entry intensity for a collimated beam of nominally monochromatic light centered at a wavelength $\lambda$ and normal to both the entry and exit faces of a sample with plane parallel faces.

Standard uncertainty, $u$ : Normally used with appropriate subscripts to describe uncertainty of the result of a measurement expressed as a standard deviation.

Transmittance density, TD: Although it is entirely equivalent to optical density, this expression has been consistently used by the NIST optical filters SRM program in certificates and reports since 1970. These documents have carried the assurance that instruments designed to report absorbance will be reporting transmittance density when the reference sample (or chemical blank) is air (an empty filter holder is provided for this purpose with NIST SRM filters). 


\section{Introduction}

Transmittance and transmittance density traceability has been offered to the chemical, pharmaceutical, and biochemical sectors of the US and global economy by the National Bureau of Standards/National Institute of Standards and Technology (NBS/NIST) since 1970. Certification in the ultraviolet and visible spectral regions (UV/vis) has been supported at NBS/NIST by a focused beam "High Accuracy Spectrophotometer" [1] (HAS, 1970-1999) and a second-generation optical clone of that instrument with modernized automation (HAS II, 2000 present). During this time, the concept of traceability [2] has been refined and the transmittance community has responded with the concept of the "transfer spectrophotometer" [3] as a link in the traceability chain. Such an instrument may be a commercial spectrophotometer linked to the primary transmittance scale by means of optical filter reference materials certified on a recognized national reference spectrophotometer.

The longstanding NBS/NIST program supporting the production and recertification of UV/visible Standard Reference Material (SRM) neutral density filters [4] in cuvette-simulation holders has qualified such a transfer spectrophotometer to assume the bulk of the certification and recertification work load and extend the useful life of the HAS II. This document details the proposed transmittance density uncertainty budget (from which transmittance uncertainties may be computed) for SRM certification with the Biochemical Science Division Transfer Spectrophotometer (BSDTS). The actual uncertainty budget is subject to change as needed, and is noted on certificates and reports that accompany certified values.

It is anticipated that the program will not construct a third generation reference spectrophotometer at the end of the HAS II life cycle, but will migrate the traceability of the BSDTS to a national reference spectrophotometer participating in international Consultative Committee for Photometry and Radiometry (CCPR) key comparisons ${ }^{1}$. [5]

\section{Certification Measurements with the Transfer Spectrophotometer}

The BSDTS is a Varian Cary $6000 \mathrm{i}^{2} \mathrm{UV} / \mathrm{visible/NIR}$ spectrophotometer with specifications well documented in the commercial literature [6]. The instrument is equipped with an optional temperature-controlled sample changer with positions for six cuvettes in the sample beam and six cuvettes in the reference beam. It is operated under the NIST quality system [7], with an initial qualification followed by quarterly performance validation and annual preventive maintenance (including lamp changes) by the manufacturer.

The application software furnished with the instrument is written in an "Application Development Language" (ADL) that is also available to end users. The operational routines used for certified transmittance density and transmittance measurements have been custom

\footnotetext{
${ }^{1}$ This document includes links to websites that may have information of interest to our users. NIST does not necessarily endorse the views expressed or the facts presented on these sites. Further, NIST does not endorse any commercial products that may be advertised or available on these sites.

${ }^{2}$ Certain commercial equipment, instruments, or materials are identified in this document. Such identification does not imply recommendation or endorsement by the National Institute of Standards and Technology, nor does it imply that the products identified are necessarily the best available for the purpose.
} 
written in ADL to conform to the greatest degree possible with the measurement sequencing and timing of the HAS II. Measurements are made and recorded in the database in transmittance. Results are reported both in transmittance (T) and in "transmittance density" (TD), given as $\log (1 / \mathrm{T})$ and equivalent to "optical density." The terminology is employed to emphasize that the measurement is not blank-corrected for surface reflection. However, the certified values for transmittance density should conform to absorbance measurements on customer instruments with the empty holder furnished with the SRM serving as the blank.

All measurements are an average of three individual readings with an averaging time of 1 second each and are made at a spectral bandwidth setting of $0.8 \mathrm{~nm}$. The "reduced height" slit image is centered $15 \mathrm{~mm}$ above the base of the holder, to best approximate the illumination of the HAS II beam. SRM filter sets each include three filters of different nominal transmittance level. A single filter set is measured during each run, with the filters placed in positions 2, 4, and 6 in the order of increasing transmittance. Position 1 is used to determine the transmittance density baseline with the reference position empty and an empty filter holder in the sample position. Position 1 is used again at the end of the run as a control to record the quality of the zero of transmittance density.

The "native" measurement position of the Cary beam is $20 \mathrm{~mm}$ above the base, and a "sample lifter" is built into the sample carriage to enable easy extraction of cuvettes. This device is used to raise the samples $5 \mathrm{~mm}$ to the certification position. The empty sample holder in Position 1 has had the top milled off to be flush with the surface of the sample carriage when the vertical position of the samples centers the optical beam as described above. The size and position of the beam is matched as closely as possible to that of the HAS II to minimize the confounded effects of beam geometry and filter heterogeneity in establishing traceability.

Data acquisition begins by taking the transmittance baseline in position 1 for all 5 wavelengths (SRM 930, SRM 1930, and SRM 2930) or 10 wavelengths (SRM 2031). The measurement proceeds by advancing to position 2, recording the transmittance at each of the 5 or 10 wavelengths, and repeating the process twice, for a total of three one-second measurements at each wavelength. This process is repeated for the other two filter-containing positions and for the empty control position. The average values of the three transmittance readings for each filter and wavelength are transmitted to the master database. The complete raw data set is retained, identified by set number and date, in addition to the reduced information transferred to the database.

The central certified values reported on new material certificates or on Reports of Recertification for returned materials represent the averages of the three transmittance measurements and the transmittance densities calculated from these average transmittances. The uncertainties reported for each filter and wavelength represent modifications of the legacy uncertainties given for HAS II measurements to include additional uncertainty resulting from the traceability of the transfer instrument to the reference instrument.

\section{Traceability Measurements}

A sequence of measurements has been made to evaluate uncertainty components resulting from the traceability of the transfer instrument based on NIST guidelines [8]. Traceability is here chosen to be provided by including uncorrected bias in the measurement uncertainty rather than 
by bias correction [9]. The expanded uncertainty $\left(U_{95}\right)$ for a certified value at a given wavelength for a given nominal transmittance density using the BSDTS is given by

$$
U_{95}=|\delta|+2 \sqrt{u_{x}^{2}+u_{\delta}^{2}+u_{r e f}^{2}}
$$

where $u_{x}$ is the standard uncertainty of a single transfer instrument measurement determined by simple replication, $u_{r e f}$ is the combined standard uncertainty for a reference standard used to compare the transfer instrument to the stated reference spectrophotometer, and $\delta$ is the bias of the transfer instrument as determined by the average of $n_{\delta}$ determinations of the measured value minus the certified value with a sample standard deviation of $S_{\delta}$ and a standard uncertainty of

$$
u_{\delta}=\sqrt{S_{\delta}^{2} / n_{\delta}}
$$

A standard uncertainty is a good approximation to a standard deviation and is expanded by a factor which is approximately 2 due to the large number of effective degrees of freedom to yield the half-width of a $95 \%$ confidence interval. [8] The standard uncertainty in Eq. 2 is so expressed as to estimate the standard deviation of the mean of the $n_{\delta}$ measurements used to determine the average value of $\delta$. The standard uncertainty $u_{x}$ is expressed as $u_{x}=S_{x}$ although replicate measurements have gone into the determination of $S_{x}$, as discussed below. This is because a single measurement will be used by the instrument to determine each certified value (where a "measurement" is taken as the average of three readings).

In Eq. 1, $u_{r e f}$ is given as half of the original certified $U_{95}$ value for the given wavelength and filter (transmittance density), and represents the combined standard uncertainty of the certified value. The original combined standard uncertainty is thus further combined by the root-sum-of-squares with standard uncertainties for the bias determination and for a single measurement with the transfer instrument. After expansion by the factor of 2, the addition of the absolute value of the measured bias provides a symmetric expanded uncertainty for transfer measurements made without bias correction. The new $U_{95}$ for the transfer instrument approaches the original $U_{95}$ for the reference instrument in the limit as $\delta$, $u_{x}$, and $S_{\delta}$ approach zero.

The largest contribution to the right-hand side of Eq. 1 is $u_{r e f}$, the combined standard uncertainty of the certified value of a given filter (i.e. nominal transmittance) at a given wavelength using the HAS II. This uncertainty is dominated by components relating to properties of the reference materials such as heterogeneity and temporal drift, discussed elsewhere [10].

For practical and traditional reasons, all uncertainty evaluations are conducted in transmittance density and are converted to transmittance as needed. Also for traditional reasons, a single uncertainty (in transmittance density) is associated with each level, applying to all certified wavelengths. To do this, the relevant uncertainty components are computed for each wavelength and the maximum is then applied to measurements at all wavelengths as a conservative estimate for each nominal transmittance. 


\subsection{Replication Uncertainty}

One set of each of the SRM types shown in the first column of Table 1 below was measured four times on each of three days for a total of 12 measurements. The values shown in the $u_{x}$ column acquired at measurement wavelength $\lambda_{x}$ represent the maximum computed sample standard deviations of these 12 measurements over all certification wavelengths for each filter. This maximum value for each filter is conservatively used for all certification wavelengths.

Table 1. Standard uncertainty of the transfer spectrophotometer for simple replication.

\begin{tabular}{|c|c|c|c|c|}
\hline SRM & Filter & TD $_{\text {nom }}$ & $\lambda_{x}$ & $U_{x}$ \\
\hline \multirow{3}{*}{930} & $10 \%$ & 1 & $440 \mathrm{~nm}$ & 0.00004 \\
\cline { 2 - 5 } & $20 \%$ & 0.7 & $440 \mathrm{~nm}$ & 0.00006 \\
\cline { 2 - 5 } 1930 & $30 \%$ & 0.5 & $440 \mathrm{~nm}$ & 0.00004 \\
\hline \multirow{3}{*}{2930} & $1 \%$ & 2 & $465 \mathrm{~nm}$ & 0.00010 \\
\cline { 2 - 5 } & $3 \%$ & 1.5 & $440 \mathrm{~nm}$ & 0.00010 \\
\cline { 2 - 5 } & $50 \%$ & 0.3 & $590 \mathrm{~nm}$ & 0.00003 \\
\hline \multirow{3}{*}{2031} & $0.1 \%$ & 3 & $440 \mathrm{~nm}$ & 0.00040 \\
\cline { 2 - 5 } & $0.3 \%$ & 2.5 & $440 \mathrm{~nm}$ & 0.00015 \\
\hline & $90 \%$ & 0.04 & $440 \mathrm{~nm}$ & 0.00006 \\
\cline { 2 - 5 } & $10 \%$ & 1 & $340 \mathrm{~nm}$ & 0.00006 \\
\hline & $30 \%$ & 0.5 & $360 \mathrm{~nm}$ & 0.00005 \\
\hline
\end{tabular}

The SRM samples were measured in sequence four times on each day, so that removal and replacement of the samples and time separation of the four measurements was assured for each sample set. Use of the three days was intended to confound any "day" effect into the determination.

\subsection{Bias Determination}

Three independent measurements were made on each of two control sets for each of the four types of SRM on the BSDTS and on the HAS II. Pairwise data sets were then used for six determinations of bias, and the average and sample standard deviation were computed for each nominal filter and wavelength. Figure 1 shows a representative result for the nominal $10 \%$ transmitting $(\mathrm{TD} \approx 1)$ filter of SRM 930. Curiously, a statistically significant wavelength dependence to the bias is observed, and the wavelength of maximum bias is reproduced in quarterly traceability validations and for the other filters measured. As before, the maximum bias over all of the wavelengths for a given filter is conservatively assigned as the bias $(\delta$ ) for all wavelengths. Table 2 gives the measured values for the bias with the corresponding sample standard deviation $\left(S_{\delta}\right)$ along with the wavelength $\left(\lambda_{\delta}\right)$ for which the maximum bias was observed. The first row of data in Table 2 represents the $635 \mathrm{~nm}$ data point of Fig. 1, and the wavelength dependence consistency across filters is supported by the preponderance of long wavelengths in the table. Since the average of six determinations is used for the bias determination, $n_{\delta}=6$ in Eq. 2 . 


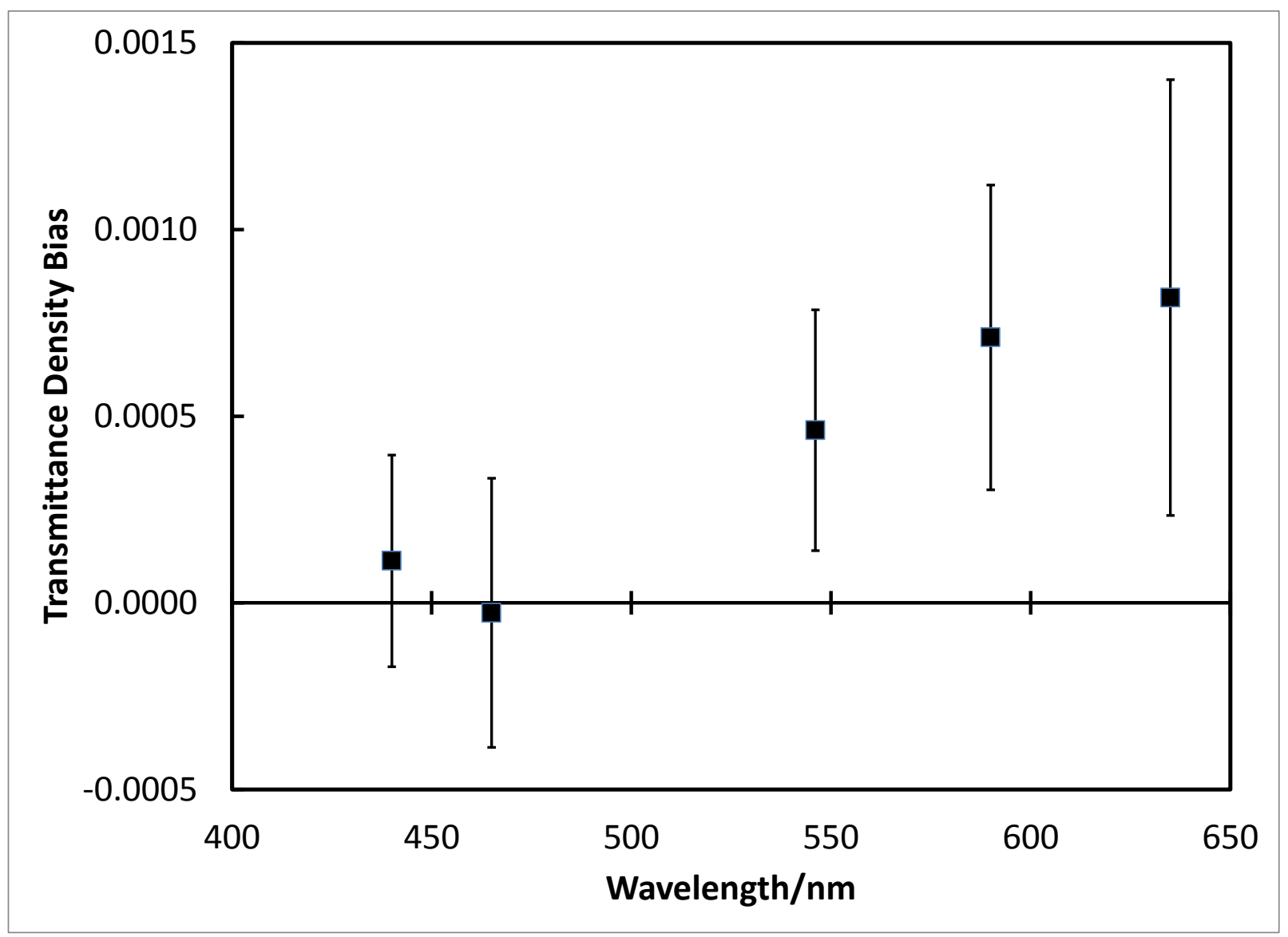

Figure 1. Transmittance density bias as a function of wavelength for the nominal $10 \%$ transmitting filter of SRM 930.

\subsection{Transfer Spectrophotometer Uncertainties}

Table 3 combines the uncertainty components of Eqs. 1 and 2 from Tables 1 and 2 with the $u_{r e f}$ value characteristic of certification on the HAS II and shows the computed value of the expanded uncertainty for transmittance density for SRMs certified on the BSDTS. NIST certificates and reports for these SRMs include tables of certified values for both transmittance density and for regular spectral transmittance. The final transmittance density uncertainties are used to compute the final uncertainties for certified transmittances through the relationship derived by differentiating the equation relating transmittance density to transmittance, $T D=-\log (\mathrm{T})$.

$$
U_{95}(T) T=U_{95} T D \times \ln (10)
$$

The uncertainties for transmittance are individually computed for each wavelength of certification using the actual measured transmittance rather than the nominal transmittance. Thus the certified transmittance tables in NIST certificates and reports shows the uncertainties varying with transmittance over the certified wavelengths whereas the transmittance density tables quote the same uncertainty for each wavelength for a given filter. 
Table 2. The bias determinations for the BSDTS with respect to the HAS II.

\begin{tabular}{|c|c|c|c|c|c|}
\hline SRM & Filter & TD $_{\text {nom }}$ & $\lambda_{\delta}$ & $\delta$ & $S_{\delta}$ \\
\hline \multirow{3}{*}{930} & $10 \%$ & 1 & 635 & 0.00082 & 0.00058 \\
\cline { 2 - 6 } & $20 \%$ & 0.7 & 590 & 0.00053 & 0.00018 \\
\cline { 2 - 6 } & $30 \%$ & 0.5 & 635 & 0.00041 & 0.00021 \\
\hline \multirow{4}{*}{2930} & $1 \%$ & 2 & 635 & 0.00196 & 0.00119 \\
\cline { 2 - 6 } & $3 \%$ & 1.5 & 635 & 0.00121 & 0.00053 \\
\cline { 2 - 6 } & $50 \%$ & 0.3 & 590 & 0.00035 & 0.00007 \\
\hline \multirow{3}{*}{2031} & $0.1 \%$ & 3 & 635 & 0.00238 & 0.00152 \\
\cline { 2 - 6 } & $0.3 \%$ & 2.5 & 635 & 0.00215 & 0.00083 \\
\cline { 2 - 6 } & $90 \%$ & 0.04 & 546 & 0.00033 & 0.00015 \\
\hline & $10 \%$ & 1 & 500 & 0.00179 & 0.00046 \\
\cline { 2 - 6 } & $30 \%$ & 0.5 & 590 & 0.00091 & 0.00007 \\
\hline
\end{tabular}

Table 3. Computation of the expanded uncertainty for the certification of transmittance density of NIST SRMs with the BSDTS using Eq. 1.

\begin{tabular}{|c|c|c|c|c|c|c|c|}
\hline SRM & Filter & $T D_{\text {nom }}$ & $u_{X}$ & $u_{\delta}$ & $u_{\text {ref }}$ & $\delta$ & $\boldsymbol{U}_{95}$ \\
\hline \multirow{3}{*}{930} & $10 \%$ & 1.0 & 0.00004 & 0.00024 & 0.00120 & 0.0008 & 0.0033 \\
\hline & $20 \%$ & 0.7 & 0.00006 & 0.00007 & 0.00115 & 0.0005 & 0.0028 \\
\hline & $30 \%$ & 0.5 & 0.00004 & 0.00009 & 0.00115 & 0.0004 & 0.0027 \\
\hline \multirow{3}{*}{1930} & $1 \%$ & 2.0 & 0.00010 & 0.00049 & 0.00275 & 0.0020 & 0.0076 \\
\hline & $3 \%$ & 1.5 & 0.00010 & 0.00022 & 0.00270 & 0.0012 & 0.0066 \\
\hline & $50 \%$ & 0.3 & 0.00003 & 0.00003 & 0.00100 & 0.0003 & 0.0023 \\
\hline \multirow{3}{*}{2930} & $0.1 \%$ & 3.0 & 0.00040 & 0.00062 & 0.00905 & 0.0024 & 0.0205 \\
\hline & $0.3 \%$ & 2.5 & 0.00015 & 0.00034 & 0.00450 & 0.0021 & 0.0112 \\
\hline & $90 \%$ & 0.04 & 0.00006 & 0.00006 & 0.00100 & 0.0003 & 0.0023 \\
\hline \multirow{3}{*}{2031} & $10 \%$ & 1.0 & 0.00006 & 0.00019 & 0.00210 & 0.0018 & 0.0060 \\
\hline & $30 \%$ & 0.5 & 0.00005 & 0.00003 & 0.00195 & 0.0009 & 0.0048 \\
\hline & $90 \%$ & 0.04 & 0.00016 & 0.00004 & 0.00095 & 0.0002 & 0.0022 \\
\hline
\end{tabular}

\section{Conclusion}

The expanded uncertainties in transmittance density for the certification of four SRMs using the Biochemical Science Division Transfer Spectrophotometer have been calculated using 12 replicate measurements of control standards to evaluate the replication uncertainty of the transfer instrument and 6 independent measurements of the bias for each filter and wavelength. The conservative nature of the results is assured by using maximum values over the various wavelengths for each transmittance density and by choosing to evaluate the symmetric uncertainty interval for uncorrected transmittance density measurements. The numerical uncertainties of Table 3 are illustrative of the process, but are subject to change as a result of future experimental results. Transmittance uncertainties are computed from the transmittance density uncertainties using the known relationship between the scales. 
The inflation of the final uncertainties by the use of the transfer instrument is expected to be acceptable for the bulk of applications in the user community. Certification using the HAS II is available at specified times and for a cost commensurate with the loss of economy of scale.

\section{Acknowledgement}

The authors are grateful to David L. Duewer of the Analytical Chemistry Division for many helpful discussions and for careful review of the manuscript. 


\section{References}

[1] R. Mavrodineanu, An Accurate Spectrophotometer for Measuring the Transmittance of Solid and Liquid Materials, J. Res. NBS 76A, 405 (1972).

[2] See http://www.nist.gov/traceability/nist_traceability_policy_external.cfm with links and references therein.

[3] See, for instance, "Technical Specifications for Certification of Spectrophotometric NTRMs," NIST Special Publication 260-140 (2000), pages 7 - 9. Can be downloaded from: http://ts.nist.gov/MeasurementServices/ReferenceMaterials/upload/SP260-140.PDF

[4] Find descriptions of SRM 930, SRM 1930, SRM 2930, and SRM 2031 at http://www.nist.gov/mml/biochemical/bioassay/optical_filters_program.cfm

[5] Gaël Obein and Jean Bastie, Report on the CCPR Key Comparison K6: Spectral regular transmittance, Metrologia 46, 02002 (2009). Can be downloaded from: http://iopscience.iop.org/0026-1394/46/1A/02002 .

[6] Available on line at: http://www.chem.agilent.com/Library/specifications/Public/si-0188.pdf .

[7] NIST Quality System, QM-I (http://www.nist.gov/qualitysystem/upload/NIST-QM-I-V6-NonControlled.pdf ); Biochemical Sciences Division Quality Manual, QM-II; Bioassay Methods Group Technical Procedure 631.02 .09

[8] Barry N. Taylor and Chris E. Kuyatt, Guidelines for Evaluating and Expressing the Uncertainty of NIST Measurement Results, NIST Technical Note 1297, U.S. Government Printing Office, Washington (1993).

[9] Bertil Magnusson \& Stephen L. R. Ellison, Treatment of uncorrected measurement bias in uncertainty estimation for chemical measurements, Anal. Bioanal. Chem. 390, 201 (2008).

[10] See the discussion of uncertainty estimation for SRM 930d in: R. Mavrodineanu, R.W. Burke, J.R. Baldwin, M.V. Smith, J.D. Messman, J.C. Travis, and J.C. Colbert, Glass Filters as a Standard Reference Material for Spectrophotometry - Selection, Preparation, Certification, and Use of SRM 930 and SRM 1930, NIST Special Publication 260-116, U.S. Government Printing Office, Washington (1994), pages 60 - 62. 